PROCEEDINGS OF THE

AMERICAN MATHEMATICAL SOCIETY

Volume 136, Number 6, June 2008, Pages 2113-2123

S 0002-9939(08)09288-5

Article electronically published on February 14, 2008

\title{
DIRICHLET-NEUMANN-IMPEDANCE BOUNDARY VALUE PROBLEMS ARISING IN RECTANGULAR WEDGE DIFFRACTION PROBLEMS
}

\author{
L. P. CASTRO AND D. KAPANADZE
}

(Communicated by Hart F. Smith)

\begin{abstract}
Boundary value problems originated by the diffraction of an electromagnetic (or acoustic) wave by a rectangular wedge with faces of possible different kinds are analyzed in a Sobolev space framework. The boundary value problems satisfy the Helmholtz equation in the interior (Lipschitz) wedge domain, and are also subject to different combinations of boundary conditions on the faces of the wedge. Namely, the following types of boundary conditions will be under study: Dirichlet-Dirichlet, Neumann-Neumann, Neumann-Dirichlet, Impedance-Dirichlet, and Impedance-Neumann. Potential theory (combined with an appropriate use of extension operators) leads to the reduction of the boundary value problems to integral equations of Fredholm type. Thus, the consideration of single and double layer potentials together with certain reflection operators originate pseudo-differential operators which allow the proof of existence and uniqueness results for the boundary value problems initially posed. Furthermore, explicit solutions are given for all the problems under consideration, and regularity results are obtained for these solutions.
\end{abstract}

\section{INTRODUCTION}

The diffraction by wedge shaped regions is a well-known and important problem in applied mathematics (cf., e.g., 1, 10, 12, 21]). Although the interest in this kind of problem has more than one century of history, recently several works have significantly increased the knowledge about the corresponding solutions and the possible spaces in which to consider these solutions. In fact, part of the recent interest is in the weak formulation of such kinds of problems, and to an eventual possibility to increase the solutions' regularity in a framework of Sobolev spaces. E.g., the works [2, 4, 5, 6, 7, 9, 16, 17, 18, 19, 20, follow this line of research, and have already worked out a correspondingly detailed mathematical analysis about several classes of diffraction problems. In addition, we refer to 15 for the physical background, history and classical references on this topic.

Received by the editors March 22, 2007.

2000 Mathematics Subject Classification. Primary 35J25; Secondary 35J05, 45E10, 47A20, 47G30, $47 \mathrm{H} 50$.

This work was supported in part by Unidade de Investigação Matemática e Aplicações of Universidade de Aveiro, and the Portuguese Science Foundation (FCT-Fundação para a Ciência e a Tecnologia) through grant number SFRH/BPD/20524/2004.

(C)2008 American Mathematical Society Reverts to public domain 28 years from publication 
In the present work, we improve the results of [17, as it concerns the knowledge about the regularity of solutions to the wedge Dirichlet-Dirichlet, NeumannNeumann, and Neumann-Dirichlet problems (cf. the next section for the precise formulation). In fact, in contrast with [17] where the unique solutions to these three problems were obtained in $H^{1}$ Bessel potential spaces (based on an extensive use of the Fourier-Plancherel operator), in the present work (by other methods) the unique solutions for these problems are obtained in $H^{1+\varepsilon}$, with $\varepsilon \in[0,1 / 2)$; cf. the corresponding particular forms of Theorems 5.2, 5.3 and 5.4. In addition, the wedge diffraction problems with Dirichlet-Impedance and Neumann-Impedance boundary conditions are also treated in the present work - therefore enlarging the type of classes considered and presented in [6] once again only for $H^{1}$ spaces (cf. also the overview about known results described in [6, p. 217, Table 1]). In this way, Theorems 5.2 and 5.3 may be viewed as the main results of the present work due to their conclusions about the explicit unique solutions for the Dirichlet-Impedance and Neumann-Impedance wedge problems in $H^{1+\varepsilon}$ (with $0 \leq \varepsilon<1 / 2$ ), respectively.

It is worth mentioning that the present methods are different from the just-cited works, which were mainly based on the Wiener-Hopf method and its generalizations. The roots of our techniques may be addressed to the Green formula and pseudo-differential operators theory. More precisely, here we use a potential theory approach combined with the use of concrete extension operators, and a corresponding integral description of the problems. Therefore, this is also different from the most classical approach due to the Malyuzhinets method 13 and corresponding representations of solutions by using the so-called Sommerfeld integral transform (cf., e.g., [11, 23]). In addition, the present wedge diffraction problems present an increase of the difficulties when compared with the corresponding problems of wave diffraction by a half-plane; cf. 3]. The main difficulty is related to the different geometries of these two classes of problems. E.g., the above mentioned concrete extension operators (which are somehow generated by wedge geometry) - being crucial in the present method (and leading to important consequences in the smoothness space orders) - do not play any role in the half-plane geometry problems.

\section{Basic notation AND FORMulation of the PROBlems}

As usual, $\mathcal{S}\left(\mathbb{R}^{n}\right)$ denotes the Schwartz space of all rapidly vanishing functions and $\mathcal{S}^{\prime}\left(\mathbb{R}^{n}\right)$ the dual space of tempered distributions on $\mathbb{R}^{n}$. The Bessel potential space $H^{s}\left(\mathbb{R}^{n}\right)$, with $s \in \mathbb{R}$, is formed by the elements $\varphi \in \mathcal{S}^{\prime}\left(\mathbb{R}^{n}\right)$ such that

$$
\|\varphi\|_{H^{s}\left(\mathbb{R}^{n}\right)}=\left\|\mathcal{F}^{-1}\left(1+|\xi|^{2}\right)^{s / 2} \cdot \mathcal{F} \varphi\right\|_{L_{2}\left(\mathbb{R}^{n}\right)}
$$

is finite. As the notation indicates, $\|\cdot\|_{H^{s}\left(\mathbb{R}^{n}\right)}$ is a norm for the space $H^{s}\left(\mathbb{R}^{n}\right)$ which makes it a Banach space. Here, $\mathcal{F}=\mathcal{F}_{x \mapsto \xi}$ denotes the Fourier transformation in $\mathbb{R}^{n}$.

For a given domain $\mathcal{D}$ on $\mathbb{R}^{n}$, we denote by $\widetilde{H}^{s}(\mathcal{D})$ the closed subspace of $H^{s}\left(\mathbb{R}^{n}\right)$ whose elements have supports in $\overline{\mathcal{D}}$, and $H^{s}(\mathcal{D})$ denotes the space of generalized functions on $\mathcal{D}$ which have extensions into $\mathbb{R}^{n}$ that belong to $H^{s}\left(\mathbb{R}^{n}\right)$. The space $\widetilde{H}^{s}(\mathcal{D})$ is endowed with the subspace topology, and on $H^{s}(\mathcal{D})$ we introduce the norm of the quotient space $H^{s}\left(\mathbb{R}^{n}\right) / \widetilde{H}^{s}\left(\mathbb{R}^{n} \backslash \overline{\mathcal{D}}\right)$. Obviously, these definitions are valid for $L_{2}$ spaces. Note that the spaces $H^{0}\left(\mathbb{R}_{+}^{n}\right)$ and $\widetilde{H}^{0}\left(\mathbb{R}_{+}^{n}\right)$ can be identified, and we will denote them by $L_{2}\left(\mathbb{R}_{+}^{n}\right)$. 
Let $\Omega:=\left\{x:=\left(x_{1}, x_{2}\right) \in \mathbb{R}^{2}: x_{1}>0, x_{2}>0\right\}, \Gamma_{1}:=\left\{\left(x_{1}, 0\right): x_{1} \in \mathbb{R}\right\}$ and $\Gamma_{2}:=\left\{\left(0, x_{2}\right): x_{2} \in \mathbb{R}\right\}$. Further, let $\Gamma_{1,+}:=\left\{\left(x_{1}, 0\right): x_{1} \in \mathbb{R}_{+}\right\}, \Gamma_{2,+}:=\left\{\left(0, x_{2}\right):\right.$ $\left.x_{2} \in \mathbb{R}_{+}\right\}$and $\partial \Omega:=\Gamma_{1,+} \cup \Gamma_{2,+} \cup\{(0,0)\}$. Denote by $n_{1}=\overrightarrow{(0,-1)}, n_{2}=\overrightarrow{(-1,0)}$ the unit normal vectors on $\Gamma_{1}$ and $\Gamma_{2}$, respectively.

We are interested in studying the problem of existence and uniqueness of an element $u \in H^{1+\varepsilon}(\Omega), 0 \leq \varepsilon<1$, such that

$$
\begin{aligned}
& \left(\Delta+k^{2}\right) u=0 \text { in } \Omega, \\
& c_{j}\left[\partial_{n_{j}} u\right]_{\Gamma_{j,+}}^{+}-d_{j}[u]_{\Gamma_{j,+}^{+}}^{+}=h_{j} \text { on } \Gamma_{j,+},
\end{aligned}
$$

where the wave number $k \in \mathbb{C} \backslash \mathbb{R}$ is given, as well as the constants $c_{j}, d_{j} \in \mathbb{C}$ $(j=1,2)$. In addition, $\Delta$ stands for the Laplace operator, and the Dirichlet and Neumann traces on $\Gamma_{j,+}$ are denoted by $[u]_{\Gamma_{j,+}}^{+}$and $\left[\partial_{n_{j}} u\right]_{\Gamma_{j,+}}^{+}$, respectively. Note that the Dirichlet type condition in (2.2) can be understood in the trace sense, while the Neumann type condition is understood in the distributional sense (cf. 14]). Finally, for $j=1,2$, the elements $h_{j} \in H^{-1 / 2+\varepsilon}\left(\Gamma_{j,+}\right)$, if $c_{j} \neq 0$, and $h_{j} \in H^{1 / 2+\varepsilon}\left(\Gamma_{j,+}\right)$, if $c_{j}=0$, respectively, are arbitrarily given since the dependence on the data is to be studied for well-posedness.

From (2.2) let us single out the following three boundary conditions:

$$
\begin{aligned}
{\left[\partial_{n} u\right]_{\Gamma_{1,+}}^{+}-p[u]_{\Gamma_{1,+}}^{+}=h_{1} \text { on } \Gamma_{1,+}, } & {[u]_{\Gamma_{2,+}}^{+}=h_{2} \text { on } \Gamma_{2,+}, } \\
{\left[\partial_{n} u\right]_{\Gamma_{1,+}}^{+}-p[u]_{\Gamma_{1,+}}^{+}=h_{1} \text { on } \Gamma_{1,+}, } & {\left[\partial_{n} u\right]_{\Gamma_{2,+}}^{+}=h_{2} \text { on } \Gamma_{2,+}, } \\
{[u]_{\Gamma_{1,+}}^{+}=h_{1} \text { on } \Gamma_{1,+}, } & {[u]_{\Gamma_{2,+}}^{+}=h_{2} \text { on } \Gamma_{2,+}, }
\end{aligned}
$$

where $p \in \mathbb{C}$. Note that the conditions on $\Gamma_{1,+}$ and $\Gamma_{2,+}$ can be interchanged, therefore leading to corresponding problems which can be treated in an entire corresponding way as those (and therefore such a detailed exposition will be omitted here).

From now on we will refer to:

- Problem $\mathcal{P}_{\text {I-D }}$ as the problem characterized by (2.1) and (2.3);

- Problem $\mathcal{P}_{\mathrm{I}-\mathrm{N}}$ as the one characterized by (2.1), (2.4), and the compatibility condition $h_{1}(x)+h_{2}\left(e^{i \frac{\pi}{2}} x\right) \in r_{\Gamma_{1,+}} \widetilde{H}^{-1 / 2+\varepsilon}\left(\Gamma_{1,+}\right)$;

- Problem $\mathcal{P}_{D-D}$ as the one characterized by (2.1), (2.5), and the compatibility condition $h_{1}(x)-h_{2}\left(e^{i \frac{\pi}{2}} x\right) \in r_{\Gamma_{1,+}} \widetilde{H}^{1 / 2+\varepsilon}\left(\Gamma_{1,+}\right)$.

For $\Sigma=\Gamma_{1,+}, \Gamma_{2,+}, \mathbb{R}_{ \pm}$(with $\mathbb{R}_{ \pm}=\{x \in \mathbb{R}: \pm x>0\}$ ), here and below we denote by $r_{\Sigma}$ the restriction operator to $\Sigma$ (defined in corresponding tilda Bessel potential spaces $\left.\widetilde{H}^{s}(\Sigma)\right)$. As for the compatibility conditions stated above, since $r_{\Sigma} \widetilde{H}^{s}(\Sigma)=$ $H^{s}(\Sigma)$ if and only if $s \in(-1 / 2,1 / 2)$, it follows that for the proposed values of $\varepsilon$ the compatibility condition included in Problem $\mathcal{P}_{\text {I-N }}$ is an additional restriction only for $\varepsilon=0$. In the case of Problem $\mathcal{P}_{D-D}$, the mentioned compatibility condition is therefore an efective restriction on the data for all the values of $\varepsilon \in[0,1)$.

We point out that Problem $\mathcal{P}_{I-N}$ includes the Neumann-Neumann problem $\left(\mathcal{P}_{N-N}\right)$, in the particular case of $p=0$, as well as the Problem $\mathcal{P}_{I-D}$ includes the Neumann-Dirichlet problem $\left(\mathcal{P}_{\mathrm{N}-\mathrm{D}}\right)$. 


\section{Uniqueness of SOLUtion FOR THE homogeneOUS PROBLEMS IN $H^{1}$ SPACES}

In this section we will present conditions which will guarantee the uniqueness of the solution for each of the corresponding homogeneous problems (in the case of $\varepsilon=0)$.

Theorem 3.1. If $\varepsilon=0$, and one of the following situations holds:

(a) $(\Re \mathrm{e} k)(\Im \mathrm{m} k)>0, \quad \Im \mathrm{m} p \geq 0$,

(b) $(\Re \mathrm{e} k)(\Im \mathrm{m} k)<0, \quad \Im \mathrm{m} p \leq 0$,

(c) $|\Im \mathrm{m} k| \geq \mid \Re$ e $k \mid, \quad \Re$ e $p \leq 0$,

(d) $\Re$ e $k=0, \Im$ m $p \neq 0$,

(e) $\Re \mathrm{e} k \neq 0, \Im \mathrm{m} p \neq 0,(\Im \mathrm{m} k)^{2}-(\Re \mathrm{e} k)^{2}+2(\Re \mathrm{e} k)(\Im \mathrm{m} k) \frac{\Re \mathrm{e} p}{\Im \mathrm{m} p} \geq 0$

then the homogeneous problems $\mathcal{P}_{I-D}$ and $\mathcal{P}_{I-N}\left(h_{1}=h_{2}=0\right)$ have only the trivial solution $u=0$ in the space $H^{1}(\Omega)$.

Proof. Let $R$ be a sufficiently large positive number and $B(R)$ be the disk centered at the origin with radius $R$. Set $\Omega_{R}:=\Omega \cap B(R)$. Note that the domain $\Omega_{R}$ has a piecewise smooth boundary

$$
S_{R}:=(\partial B(R) \cap \Omega) \cup\left(\Gamma_{1,+} \cap B(R)\right) \cup\left(\Gamma_{2,+} \cap B(R)\right),
$$

and denote by $n(x)$ the outward unit normal vector at the non-singular point $x \in$ $S_{R}$.

Let $u$ be a solution of the homogeneous problem. Then the Green formula (see, e.g., [22]) for $u$ and its complex conjugate $\bar{u}$ in the domain $\Omega_{R}$ yields

$$
\int_{\Omega_{R}}\left[|\nabla u|^{2}-k^{2}|u|^{2}\right] d x=\int_{S_{R}} \partial_{n} u \bar{u} d S_{R}
$$

From (3.1) we obtain

$$
\begin{aligned}
& \int_{\Omega_{R}}\left[|\nabla u|^{2}-k^{2}|u|^{2}\right] d x \\
& =\int_{\Gamma_{1,+} \cap B(R)}\left[\partial_{n} u\right]^{+}[\bar{u}]^{+} d x+\int_{\Gamma_{2,+} \cap B(R)}\left[\partial_{n} u\right]^{+}[\bar{u}]^{+} d x+\int_{\partial B(R) \cap \Omega}\left[\partial_{n} u\right]^{+}[\bar{u}]^{+} d S \\
& =p \int_{\mathbb{R}_{+} \cap B(R)}\left|[u]^{+}\right|^{2} d x+\int_{\partial B(R) \cap \Omega}\left[\partial_{n} u\right]^{+}[\bar{u}]^{+} d S
\end{aligned}
$$

Note that, since $\Im \mathrm{m} k \neq 0$, the integral $\int_{\partial B(R) \cap \Omega}\left[\partial_{n} u\right]^{+}[\bar{u}]^{+} d S$ tends to 0 as $R \rightarrow \infty$. Indeed, in $(R, \phi)$ polar coordinates we have

$$
\int_{\partial B(R) \cap \Omega}\left[\partial_{n} u\right]^{+}[\bar{u}]^{+} d S=R \int_{0}^{\frac{\pi}{2}} \partial_{n} u \bar{u} d \phi=R \lim _{\delta_{1}, \delta_{2} \rightarrow 0+} \int_{\delta_{1}}^{\frac{\pi}{2}-\delta_{2}} \partial_{n} u \bar{u} d \phi
$$

and we take into account that the solution $u \in H^{1}(\Omega)$ of the Helmholtz equation exponentially vanishes at infinity in the sector $\phi \in\left(\delta_{1}, \frac{\pi}{2}-\delta_{2}\right)$. Therefore passing to the limit as $R \rightarrow \infty$ in (3.2) it follows that

$$
\int_{\Omega}\left[|\nabla u|^{2}-k^{2}|u|^{2}\right] d x=p \int_{\mathbb{R}_{+}}\left|[u]^{+}\right|^{2} d x
$$


Separating the real and imaginary parts of the last identity, we obtain

$$
\begin{aligned}
& \int_{\Omega}\left[|\nabla u|^{2}+\left((\Im \mathrm{m} k)^{2}-(\Re \mathrm{e} k)^{2}\right)|u|^{2}\right] d x=\Re \mathrm{e} p \int_{\mathbb{R}_{+}}\left|[u]^{+}\right|^{2} d x ; \\
& \quad-2(\Re \mathrm{e} k)(\Im \mathrm{m} k) \int_{\Omega}|u|^{2} d x=\Im \mathrm{m} p \int_{\mathbb{R}_{+}}\left|[u]^{+}\right|^{2} d x .
\end{aligned}
$$

Thus, for each of the conditions (a)-(e), it follows from the last two identities that $u=0$ in $\Omega$.

We reinforce that the conditions in Theorem 3.1 also include the analysis of the Neumann-Dirichlet case: $p=0$. In addition, in this particular case the above reasoning simplifies considerably, and it is easy to see that without restriction conditions the corresponding homogeneous problem has only the trivial solution. Moreover, an analogous result is true for the problems $\mathcal{P}_{D-D}$ and $\mathcal{P}_{\mathrm{N}-\mathrm{N}}$ :

Theorem 3.2. Let $\varepsilon=0$. Then the homogeneous problems $\mathcal{P}_{D-D}$ and $\mathcal{P}_{N-N}$ have only the trivial solution $u=0$ in the space $H^{1}(\Omega)$.

The proof of this result is analogous to the proof of Theorem 3.1, and therefore is omitted here.

\section{The Fundamental SOlution and potentials}

Let us denote the standard fundamental solution of the Helmholtz equation (in two dimensions) by

$$
\mathcal{K}(x):=-\frac{i}{4} H_{0}^{(1)}(k|x|),
$$

where $H_{0}^{(1)}(k|x|)$ is the Hankel function of the first kind of order zero (cf. [8, §3.4]). Furthermore, we introduce the single and double layer potentials on $\Gamma_{j}$ :

$$
\begin{aligned}
V_{j}(\psi)(x) & =\int_{\Gamma_{j}} \mathcal{K}(x-y) \psi(y) d_{y} \Gamma_{j}, \quad x \notin \Gamma_{j}, \\
W_{j}(\varphi)(x) & =\int_{\Gamma_{j}}\left[\partial_{n_{j}(y)} \mathcal{K}(x-y)\right] \varphi(y) d_{y} \Gamma_{j}, \quad x \notin \Gamma_{j},
\end{aligned}
$$

where $j=1,2$ and $\psi, \varphi$ are density functions. Note that for $j=1$ we will sometimes write $\mathbb{R}$ instead of $\Gamma_{1}$. In this case, for example, the single layer potential defined above has the form

$$
V_{1}(\psi)\left(x_{1}, x_{2}\right)=\int_{\mathbb{R}} \mathcal{K}\left(x_{1}-y, x_{2}\right) \psi(y) d y, \quad x_{2} \neq 0 .
$$

Set $\mathbb{R}_{ \pm}^{2}:=\left\{\left(x_{1}, x_{2}\right) \in \mathbb{R}^{2}: x_{2} \gtrless 0\right\}$ and let us first consider the operators $V:=V_{1}$ and $W:=W_{1}$.

Theorem 4.1 ([3]). The single and double layer potentials $V$ and $W$ are continuous operators

$$
V: H^{s}(\mathbb{R}) \rightarrow H^{s+1+\frac{1}{2}}\left(\mathbb{R}_{ \pm}^{2}\right), \quad W: H^{s+1}(\mathbb{R}) \rightarrow H^{s+1+\frac{1}{2}}\left(\mathbb{R}_{ \pm}^{2}\right)
$$

for all $s \in \mathbb{R}$. 
Clearly, a similar result holds true for the operators $V_{2}$ and $W_{2}$.

Let us now recall some properties of the potentials introduced above. The following jump relations are well-known (cf. [3]):

$$
\begin{aligned}
& {[V(\psi)]_{\mathbb{R}}^{+}=[V(\psi)]_{\mathbb{R}}^{-}=: \mathcal{H}(\psi), \quad\left[\partial_{n} V(\psi)\right]_{\mathbb{R}}^{ \pm}=:\left[\mp \frac{1}{2} I\right](\psi),} \\
& {[W(\varphi)]_{\mathbb{R}}^{ \pm}=:\left[ \pm \frac{1}{2} I\right](\varphi), \quad\left[\partial_{n} W(\varphi)\right]_{\mathbb{R}}^{+}=\left[\partial_{n} W(\varphi)\right]_{\mathbb{R}}^{-}=: \mathcal{L}(\varphi),}
\end{aligned}
$$

where

$$
\begin{aligned}
\mathcal{H}(\psi)(z) & :=\int_{\mathbb{R}} \mathcal{K}(z-y) \psi(y) d y, \quad z \in \mathbb{R}, \\
\mathcal{L}(\varphi)(z) & :=\lim _{\mathbb{R}_{+}^{2} \ni x \rightarrow z \in \mathbb{R}} \partial_{n(x)} \int_{\mathbb{R}}\left[\partial_{n(y)} \mathcal{K}(y-x)\right] \varphi(y) d y, \quad z \in \mathbb{R},
\end{aligned}
$$

and $I$ denotes the identity operator.

In our further reasoning we will make convenient use of the even and odd extension operators defined by

$$
\ell^{e} \varphi(y)=\left\{\begin{array}{ll}
\varphi(y), & y \in \mathbb{R}_{ \pm}, \\
\varphi(-y), & y \in \mathbb{R}_{\mp}
\end{array} \quad \text { and } \quad \ell^{o} \varphi(y)= \begin{cases}\varphi(y), & y \in \mathbb{R}_{ \pm} \\
-\varphi(-y), & y \in \mathbb{R}_{\mp}\end{cases}\right.
$$

respectively.

Remark 4.2 (cf. [5]). The operators

$$
\begin{aligned}
& \ell^{e}: H^{\varepsilon+\frac{1}{2}}\left(\mathbb{R}_{ \pm}\right) \longrightarrow H^{\varepsilon+\frac{1}{2}}(\mathbb{R}), \quad \ell^{o}: r_{\mathbb{R}_{ \pm}} \widetilde{H}^{\varepsilon+\frac{1}{2}}\left(\mathbb{R}_{ \pm}\right) \longrightarrow H^{\varepsilon+\frac{1}{2}}(\mathbb{R}), \\
& \ell^{o}: H^{\varepsilon-\frac{1}{2}}\left(\mathbb{R}_{ \pm}\right) \longrightarrow H^{\varepsilon-\frac{1}{2}}(\mathbb{R}), \quad \ell^{e}: r_{\mathbb{R}_{ \pm}} \widetilde{H}^{\varepsilon-\frac{1}{2}}\left(\mathbb{R}_{ \pm}\right) \longrightarrow H^{\varepsilon-\frac{1}{2}}(\mathbb{R}),
\end{aligned}
$$

are continuous for all $\varepsilon \in[0,1 / 2)$.

Lemma 4.3. If $0 \leq \varepsilon<1 / 2$, then

$$
\begin{aligned}
& r_{\Gamma_{2}} \circ V \circ \ell^{o} \psi=0, \quad r_{\Gamma_{2}} \circ W \circ \ell^{o} \tilde{\varphi}=0, \\
& r_{\Gamma_{2}} \circ \partial_{n_{2}} V \circ \ell^{e} \tilde{\psi}=0, \quad r_{\Gamma_{2}} \circ \partial_{n_{2}} W \circ \ell^{e} \varphi=0
\end{aligned}
$$

for all $\psi \in H^{\varepsilon-\frac{1}{2}}\left(\Gamma_{1,+}\right), \tilde{\psi} \in r_{\Gamma_{1,+}} \widetilde{H}^{\varepsilon-\frac{1}{2}}\left(\Gamma_{1,+}\right), \varphi \in H^{\varepsilon+\frac{1}{2}}\left(\Gamma_{1,+}\right)$, and $\tilde{\varphi} \in$ $r_{\Gamma_{1,+}} \widetilde{H}^{\varepsilon+\frac{1}{2}}\left(\Gamma_{1,+}\right)$.

Proof. Let us first consider the operator $r_{\Gamma_{2}} \circ V \circ \ell^{o}$. We have

$$
r_{\Gamma_{2}} \circ V \circ \ell^{o} \psi=\int_{\mathbb{R}} \mathcal{K}\left(-y, x_{2}\right) \ell^{o} \psi(y) d y, \quad x_{2} \in \Gamma_{2} .
$$

Now due to Remark 4.2 and since $\mathcal{K}\left(-y, x_{2}\right)=\mathcal{K}\left(y, x_{2}\right)$ for all $y \in \mathbb{R}, x_{2} \in \Gamma_{2}$, we obtain $r_{\Gamma_{2}} \circ V \circ \ell^{\circ} \psi=0$ for all $\psi \in H^{\varepsilon-\frac{1}{2}}\left(\Gamma_{1,+}\right)$.

Similarly, we can show the remaining identities. Note that the kernels of the operators $r_{\Gamma_{2}} \circ W, r_{\Gamma_{2}} \circ \partial_{n_{2}} V$, and $r_{\Gamma_{2}} \circ \partial_{n_{2}} W$ are even, odd, and odd functions (with respect to $y$ ), respectively.

Note that analogous results are valid for the operators $V_{2}$ and $W_{2}$. 
5. Solutions of the Problems

WITHIN A SET OF SMOOTHNESS SPACE ORDERS

Let us consider a double layer potential,

$$
u(x)=W \ell^{o} \varphi, \quad \varphi \in r_{\Gamma_{1,+}} \widetilde{H}^{\frac{1}{2}+\varepsilon}\left(\Gamma_{1,+}\right) .
$$

Then due to Lemma 4.3 we have $[u]^{+}=0$ on $\Gamma_{2}$, while on $\Gamma_{1}$ we obtain

$$
[u]^{+}=\left(\mathcal{L}-\frac{p}{2} I\right) \ell^{o} \varphi=: \mathcal{A}\left(\ell^{o} \varphi\right)
$$

Note that the operator $\mathcal{A}$ is a pseudo-differential operator with a symbol $\sigma(\mathcal{A})\left(\xi_{1}\right):=$ $\sigma(\mathcal{L})\left(\xi_{1}\right)-p / 2$, i.e.,

$$
\mathcal{A}=\mathcal{F}_{\xi_{1} \rightarrow x_{1}}^{-1} \sigma(\mathcal{A})\left(\xi_{1}\right) \mathcal{F}_{y \rightarrow \xi_{1}}
$$

here, by $\sigma(\mathcal{L})\left(\xi_{1}\right)$ we denote the complete symbol of the pseudo-differential operator $\mathcal{L}$. It is known (cf. [3]) that

$$
\sigma(\mathcal{L})\left(\xi_{1}\right)=-\frac{i w\left(\xi_{1}\right)}{2}
$$

where $w=w\left(\xi_{1}\right):=\left(a^{2}+b^{2}\right)^{\frac{1}{4}}\left(\cos \frac{\alpha}{2}+i \sin \frac{\alpha}{2}\right)$,

$$
\begin{aligned}
& a=a\left(\xi_{1}\right):=(\Re \mathrm{e} k)^{2}-(\Im \mathrm{m} k)^{2}-\xi_{1}^{2}, \\
& b:=2(\Re \mathrm{e} k)(\Im \mathrm{m} k)
\end{aligned}
$$

and

$$
\alpha:=\left\{\begin{array}{lll}
\arctan \frac{b}{|a|} & \text { if } \quad a>0, b>0, \\
\frac{\pi}{2} & \text { if } \quad a=0, b>0, \\
\pi-\arctan \frac{b}{|a|} & \text { if } \quad a<0, b>0, \\
\pi & \text { if } \quad b=0, \\
2 \pi-\arctan \frac{|b|}{|a|} & \text { if } \quad a>0, b<0, \\
\frac{3 \pi}{2} & \text { if } \quad a=0, b<0, \\
\pi+\arctan \frac{|b|}{|a|} & \text { if } \quad a<0, b<0 .
\end{array}\right.
$$

Lemma 5.1. Let one of the conditions (a)-(e) of Theorem 3.1 be satisfied. Then

$$
\sigma(\mathcal{A})\left(\xi_{1}\right) \neq 0
$$

for all $\xi_{1} \in \mathbb{R}$.

Proof. It is sufficient to show that $w\left(\xi_{1}\right) \neq i p=-\Im \mathrm{m} p+i \Re \mathrm{e} p$ for all $\xi_{1} \in \mathbb{R}$ provided that one of the conditions (a)-(e) of Theorem 3.1 is satisfied.

If $(\Re \mathrm{e} k)(\Im \mathrm{m} k)>0$, then from (5.2) we have that $\arg w\left(\xi_{1}\right) \in\left(0, \frac{\pi}{2}\right)$, i.e., $\Re \mathrm{e} w\left(\xi_{1}\right)>0$ for all $\xi_{1} \in \mathbb{R}$, while condition (a) gives us $-\Im \mathrm{m} p \leq 0$, and therefore $w\left(\xi_{1}\right) \neq i p$. Case (b) is treated similarly. Condition (c) also gives us the desired result since by definition $\Im \mathrm{m} w>0$. If $\Re \mathrm{e} k=0$, then $\arg w\left(\xi_{1}\right)=\pi / 2$ and therefore $w\left(\xi_{1}\right) \neq i p$ since $\Im \mathrm{m} p \neq 0$.

For the remaining case let us mention that $w\left(\xi_{1}\right) \neq 0$, for all $\xi_{1} \in \mathbb{R}$; cf. (5.2). Now assuming $w\left(\xi_{1}\right)=i p$, it therefore follows that $w^{2}\left(\xi_{1}\right)=-p^{2}$. Separating the real and imaginary parts (in this last identity), we obtain

$$
-\left(\Re \mathrm{e} p^{+}\right)^{2}+\left(\Im \mathrm{m} p^{+}\right)^{2}=(\Re \mathrm{e} k)^{2}-(\Im \mathrm{m} k)^{2}-\xi_{1}^{2}
$$


and

$$
-\left(\Re \mathrm{e} p^{+}\right)\left(\Im \mathrm{m} p^{+}\right)=(\Re \mathrm{e} k)(\Im \mathrm{m} k) .
$$

Then we have

$$
(\Im \mathrm{m} k)^{2}-(\Re \mathrm{e} k)^{2}+2(\Re \mathrm{e} k)(\Im \mathrm{m} k) \frac{\Im \mathrm{m} p^{+}}{\Re \mathrm{e} p^{+}}=-\left(\Im \mathrm{m} p^{+}\right)^{2}-\left(\Re \mathrm{e} p^{+}\right)^{2}-\xi_{1}^{2}<0,
$$

which contradicts the corresponding condition in (e).

Theorem 5.2. Let one of the conditions (a)-(e) of Theorem 3.1 be satisfied. If the boundary data satisfy the conditions

$$
\left(h_{1}, h_{2}\right) \in H^{-\frac{1}{2}+\varepsilon}\left(\Gamma_{1,+}\right) \times H^{\frac{1}{2}+\varepsilon}\left(\Gamma_{2,+}\right)
$$

for $0 \leq \varepsilon<\frac{1}{2}$, then the Problem $\mathcal{P}_{\text {I-D }}$ has a unique solution $u \in H^{1+\varepsilon}(\Omega)$, which is representable in the form

$$
u=W_{1} \mathcal{A}^{-1}\left(\ell^{o} g\right)+2 W_{2}\left(\ell h_{2}\right)
$$

where $\ell h_{2}$ is any extension of $h_{2}$ such that $\ell h_{2} \in H^{\frac{1}{2}+\varepsilon}\left(\Gamma_{2}\right)$ and

$$
g:=h_{1}-2\left[\partial_{n} W_{2}\left(\ell h_{2}\right)\right]_{\Gamma_{1,+}}^{+}+2 p\left[W_{2}\left(\ell h_{2}\right)\right]_{\Gamma_{1,+}}^{+} .
$$

Proof. Let $0 \leq \varepsilon<\frac{1}{2}$, and let one of the conditions (a)-(e) of Theorem 3.1 be satisfied. Then, due to Theorem 3.1, we only need to show that $u$ represented in (5.3) is a solution of the Problem $\mathcal{P}_{\text {I-D }}$. Clearly, it is a solution of the Helmholtz equation in $\Omega$. Therefore, the boundary conditions remain to be checked.

Observe first that Lemma 5.1 implies the invertibility of the operator

$$
\mathcal{A}: H^{\frac{1}{2}+\varepsilon}\left(\Gamma_{1}\right) \rightarrow H^{-\frac{1}{2}+\varepsilon}\left(\Gamma_{1}\right)
$$

and

$$
\mathcal{A}^{-1}=\mathcal{F}_{\xi_{1} \rightarrow x_{1}}^{-1}\left[\sigma(\mathcal{A})\left(\xi_{1}\right)\right]^{-1} \mathcal{F}_{y \rightarrow \xi_{1}} .
$$

Moreover, since $\sigma(\mathcal{A})$ is an even function, then

$$
\mathcal{A} J=J \mathcal{A},
$$

where $J$ is a reflection operator given by the rule

$$
J \varphi(y)=\varphi(-y), \quad \text { for all } y \in \mathbb{R} .
$$

Thus, if $g \in H^{-\frac{1}{2}+\varepsilon}\left(\Gamma_{1,+}\right)$, then $\mathcal{A}^{-1}\left(\ell^{o} g\right)$ is an odd function, and therefore

$$
\mathcal{A}^{-1}\left(\ell^{o} g\right)=\ell^{o} r_{\Gamma_{1,+}} \mathcal{A}^{-1}\left(\ell^{o} g\right) \text {. }
$$

This observation, together with the jump relations (4.2) and Lemma 4.3 (which holds for $\varepsilon<\frac{1}{2}$ ), yields that $[u]_{\Gamma_{2,+}}^{+}=h_{2}$.

Furthermore, by defining

$$
v:=2 W_{2}\left(\ell h_{2}\right)
$$

we have $g=h_{1}-\left(\left[\partial_{n} v\right]_{\Gamma_{1,+}}^{+}-p[v]_{\Gamma_{1,+}}^{+}\right)$, and it is clear that $g \in H^{-\frac{1}{2}+\varepsilon}\left(\Gamma_{1,+}\right)$ (cf. Theorem 4.11). Then we have

$$
\left[\partial_{n} u\right]_{\Gamma_{1,+}}^{+}-p[u]_{\Gamma_{1,+}}^{+}=r_{\Gamma_{1,+}} \mathcal{A} \mathcal{A}^{-1}\left(\ell^{o} g\right)+\left(\left[\partial_{n} v\right]_{\Gamma_{1,+}}^{+}-p[v]_{\Gamma_{1,+}}^{+}\right)=h_{1},
$$

which concludes the proof. 
Note that the last result contains a certain degree of freedom, as it concerns the choice of the concrete extension operator $\ell$. In particular, if we use the even extension operator $\ell^{e}$ instead of $\ell$ we obtain a more simple representation formula of the solution, namely

$$
u=W_{1} \mathcal{A}^{-1}\left(\ell^{o} g\right)+2 W_{2}\left(\ell^{e} h_{2}\right)
$$

where

$$
g=h_{1}+2 p\left[W_{2}\left(\ell h_{2}\right)\right]_{\Gamma_{1,+}}^{+}
$$

(cf. Lemma 4.3). For the case $p=0$, we have an obvious simplification of (5.3):

$$
u=W_{1} \mathcal{A}^{-1}\left(\ell^{o} h_{1}\right)+2 W_{2}\left(\ell^{e} h_{2}\right) .
$$

Moreover, using a single layer potential we can also rewrite $u$ as

$$
u=2 W_{2}\left(\ell^{e} h_{2}\right)-2 V_{1}\left(\ell^{o} h_{1}\right) .
$$

Theorem 5.3. Let one of the conditions (a)-(e) of Theorem 3.1 be satisfied. If the boundary data satisfy the conditions

$$
\left(h_{1}, h_{2}\right) \in H^{-\frac{1}{2}+\varepsilon}\left(\Gamma_{1,+}\right) \times H^{-\frac{1}{2}+\varepsilon}\left(\Gamma_{2,+}\right)
$$

for $0 \leq \varepsilon<\frac{1}{2}$, then the Problem $\mathcal{P}_{I-N}$ has a unique solution $u \in H^{1+\varepsilon}(\Omega)$, which is representable in the form

$$
u=W_{1} \mathcal{A}^{-1}\left(\ell^{e} g\right)-2 V_{2}\left(\ell h_{2}\right),
$$

where $\ell h_{2}$ is any extension of $h_{2}$ which belongs to $H^{-\frac{1}{2}+\varepsilon}\left(\Gamma_{2}\right)$ and

$$
g:=h_{1}+2\left[\partial_{n} V_{2}\left(\ell h_{2}\right)\right]_{\Gamma_{1,+}}^{+}-2 p\left[V_{2}\left(\ell h_{2}\right)\right]_{\Gamma_{1,+}}^{+} .
$$

Proof. The present proof is analogous to the one of Theorem 5.2 and we will just point out the differences here. Note that

$$
\left[V_{2}\left(\ell h_{2}\right)\right]_{\Gamma_{1,+}}^{+} \in r_{\Gamma_{1,+}} \widetilde{H}^{-\frac{1}{2}+\varepsilon}\left(\Gamma_{1,+}\right)
$$

and that the compatibility condition gives us $g \in r_{\Gamma_{1,+}} \widetilde{H}^{-\frac{1}{2}+\varepsilon}\left(\Gamma_{1,+}\right)$ (cf. the end of $₫ 2)$. Thus, we can apply the operator $\ell^{e}$ to $g$; cf. Remark 4.2. Then $\mathcal{A}^{-1}\left(\ell^{e} g\right)$ is an even function, and we have $r_{\Gamma_{2}} \circ \partial_{n_{2}} W_{1} \mathcal{A}^{-1}\left(\ell^{e} g\right)=0$. Therefore $\left[\partial_{n} u\right]_{\Gamma_{2,+}}=h_{2}$ and

$$
\left[\partial_{n} u\right]_{\Gamma_{1,+}}^{+}-p[u]_{\Gamma_{1,+}}^{+}=g-2\left[\partial_{n} V_{2}\left(\ell h_{2}\right)\right]_{\Gamma_{1,+}}^{+}+2 p\left[V_{2}\left(\ell h_{2}\right)\right]_{\Gamma_{1,+}}^{+}=h_{1}
$$

If we take the odd extension $\ell^{o}$ instead of $\ell$ we obtain that

$$
g=h_{1}+2\left[\partial_{n} V_{2}\left(\ell^{o} h_{2}\right)\right]_{\Gamma_{1,+}}^{+} .
$$

Note that Theorem 5.3 includes the case of the Neumann-Neumann problem ( $p=$ $0)$. In this case, by using the single layer potential, (5.5) can also be written as

$$
u=-2 V_{1}\left(\ell^{e} g\right)-2 V_{2}\left(\ell^{o} h_{2}\right) \text {. }
$$

If $\left(h_{1}, h_{2}\right) \in r_{\Gamma_{1,+}} \widetilde{H}^{-\frac{1}{2}+\varepsilon}\left(\Gamma_{1,+}\right) \times r_{\Gamma_{2,+}} \widetilde{H}^{-\frac{1}{2}+\varepsilon}\left(\Gamma_{2,+}\right)$, then the solution can be represented as

$$
u=-2 V_{1}\left(\ell^{e} h_{1}\right)-2 V_{2}\left(\ell^{e} h_{2}\right) .
$$


Theorem 5.4. If the boundary data satisfy the conditions

$$
\left(h_{1}, h_{2}\right) \in H^{\frac{1}{2}+\varepsilon}\left(\Gamma_{1,+}\right) \times H^{\frac{1}{2}+\varepsilon}\left(\Gamma_{2,+}\right)
$$

for $0 \leq \varepsilon<\frac{1}{2}$, then the Problem $\mathcal{P}_{D-D}$ has a unique solution $u \in H^{1+\varepsilon}(\Omega)$, which is representable in the form

$$
u=2 W_{1}\left(\ell^{o} g\right)+2 W_{2}\left(\ell h_{2}\right)
$$

where $\ell h_{2}$ is any extension of $h_{2}$ such that $\ell h_{2} \in H^{\frac{1}{2}+\varepsilon}\left(\Gamma_{2}\right)$ and

$$
g:=h_{1}-\left[2 W_{2}\left(\ell h_{2}\right)\right]_{\Gamma_{1,+}}^{+} .
$$

The proof of this result is very similar to the corresponding proofs presented above (and is therefore omitted here).

Note that if $\left(h_{1}, h_{2}\right) \in r_{\Gamma_{1,+}} \widetilde{H}^{\frac{1}{2}+\varepsilon}\left(\Gamma_{1,+}\right) \times r_{\Gamma_{2,+}} \widetilde{H}^{\frac{1}{2}+\varepsilon}\left(\Gamma_{2,+}\right)$, then the unique solution of the problem $\mathcal{P}_{D-D}$ can be written as

$$
u=2 W_{1}\left(\ell^{o} h_{1}\right)+2 W_{2}\left(\ell^{\circ} h_{2}\right) .
$$

\section{REFERENCES}

1. B. Budaev, Diffraction by Wedges, Pitman Research Notes in Mathematics Series 322. Harlow: Longman Scientific \& Technical (1995). MR 1413497 (98b:35024)

2. L.P. Castro and D. Kapanadze, Wave diffraction by a strip with first and second kind boundary conditions: the real wave number case, Math. Nachr., to appear, 12 pp.

3. L.P. Castro and D. Kapanadze, On wave diffraction by a half-plane with different face impedances, Math. Methods Appl. Sci. 30 (2007), 513-527. MR2298679

4. L.P. Castro and D. Natroshvili, The potential method for the reactance wave diffraction problem in a scale of spaces, Georgian Math. J. 13 (2006), 251-260. MR2252591 (2007d:35029)

5. L.P. Castro, F.-O. Speck, and F.S. Teixeira, Explicit solution of a Dirichlet-Neumann wedge diffraction problem with a strip, J. Integral Equations Appl. 5 (2003), 359-383. MR2058809 (2005m:35054)

6. L.P. Castro, F.-O. Speck, and F.S. Teixeira, On a class of wedge diffraction problems posted by Erhard Meister, Oper. Theory, Adv. Appl. 147 (2004), 213-240. MR2053691 (2005b:35039)

7. L.P. Castro, F.-O. Speck, and F.S. Teixeira, Mixed boundary value problems for the Helmholtz equation in a quadrant, Integral Equations Oper. Theory 56 (2006), 1-44. MR2256995

8. D. Colton and R. Kress, Inverse Acoustic and Electronic Scattering Theory. Berlin: SpringerVerlag (1998). MR1635980 (99c:35181)

9. M. Costabel and E. Stephan, A direct boundary integral equation method for transmission problems, J. Math. Anal. Appl. 106 (1985), 367-413. MR782799 (86f:76045)

10. J.-P. Croisille and G. Lebeau, Diffraction by an Immersed Elastic Wedge. Lecture Notes in Mathematics 1723. Berlin: Springer (1999). MR1740860 (2001e:74049)

11. A.I. Komech, N.J. Mauser, and A.E. Merzon, On Sommerfeld representation and uniqueness in scattering by wedges, Math. Methods Appl. Sci. (2) 28 (2005), 147-183. MR2110262 (2006c:35166)

12. A.K. Gautesen, Diffraction of plane waves by a wedge with impedance boundary conditions, Wave Motion 41 (2005), 239-246. MR2120170 (2005h:76073)

13. G.D. Malyuzhinets, Excitation, reflection and emission of surface waves from a wedge with given face impedances (English; Russian original), Sov. Phys., Dokl. 3 (1959), 752-755; translation from Dokl. Akad. Nauk SSSR 121 (1959), 436-439.

14. W. McLean, Strongly Elliptic Systems and Boundary Integral Equations. Cambridge: Cambridge University Press (2000). MR.1742312 (2001a:35051)

15. E. Meister, Some solved and unsolved canonical problems of diffraction theory, Lecture Notes in Math. 1285 (1987), 320-336. MR921283 (88m:35036)

16. E. Meister, F.-O. Speck, and F.S. Teixeira, Wiener-Hopf-Hankel operators for some wedge diffraction problems with mixed boundary conditions, J. Integral Equations Appl. (2) 4 (1992), 229-255. MR.1172891 (93h:47032) 
17. E. Meister, F. Penzel, F.-O. Speck, and F. S. Teixeira, Some interior and exterior boundary value problems for the Helmholtz equation in a quadrant, Proc. Roy. Soc. Edinburgh Sect. A 123 (1993), 275-294. MR1215413 (94b:35081)

18. E. Meister, F. Penzel, F.-O. Speck, and F.S. Teixeira, Two canonical wedge problems for the Helmholtz equation, Math. Methods Appl. Sci. 17 (1994), 877-899. MR1289599(95f:35049)

19. E. Meister and F.-O. Speck, Modern Wiener-Hopf methods in diffraction theory, Pitman Res. Notes Math. Ser. 216 (1989), 130-171. MR1031728 (91a:35049)

20. F. Penzel and F. S. Teixeira, The Helmholtz equation in a quadrant with Robin's conditions, Math. Methods Appl. Sci. 22 (1999), 201-216. MR.1672263 (2000a:35029)

21. A.D. Rawlins, A note on point source diffraction by a wedge, J. Appl. Math. (1) 2004 (2004), 85-89. MR2077882 (2005d:35152)

22. S.L. Sobolev, Partial Differential Equations of Mathematical Physics (English translation). New-York: Dover Publications (1989).

23. A. Sommerfeld, Mathematische Theorie der Diffraction (in German), Math. Ann. 47 (1896), 317-374. MR 1510907

Research Unit "Mathematics and Applications", Department of Mathematics, University of Aveiro, 3810-193 Aveiro, Portugal

E-mail address: castro@ua.pt

Research Unit "Mathematics and Applications", Department of Mathematics, University of Aveiro, 3810-193 Aveiro, Portugal

E-mail address: david.kapanadze@gmail.com 